\title{
Analoge Räume - zur Geschichte des komplexen Verhältnisses von Information, Wissen und Raum
}

\author{
Ein Beitrag von Thomas Hapke ${ }^{1}$ \\ Preprint, publiziert in: ABI-Technik 29 (2009) 2, 102-116.
}

\begin{abstract}
Die interdisziplinäre, 2008 in Belgien stattgefundene Konferenz "Analogous spaces - architecture and the space of information, intellect and action" präsentierte einerseits Forschungsaktivitäten zur Geschichte wissenschaftlicher Information und Kommunikation und war damit auch eine Reise zu den Anfängen der frühen Informationsgesellschaft. Andererseits vermittelt sie Informationsgeschichte mit Kultur-, Architektur- und Kunstgeschichte, indem auf diverse Analogien zwischen analogen und digitalen Räumen Bezug genommen wurde. Thematischer Ausgangspunkt war der Belgier Paul Otlet als Begründer der Dokumentation um die Wende zum 20. Jahrhundert, von dessen intellektuellem Netzwerk hier in einem Exkurs auch der Chemiker und Pionier des deutschen Informationswesens Wilhelm Ostwald besonders erwähnt wird.
\end{abstract}

Analogous spaces - on the history of the complex relation between information, knowledge and space

On the one hand the interdisciplinary conference "Analogous spaces - architecture and the space of information, intellect and action" taking place 2008 in Belgium presented research activities on the history of scholarly information and communication. With it at the same time it was a journey to the origins of the early information society. Otherwise the conference connected information history with the history of culture, architecture and art by referring various analogies between analog and digital spaces. Topical starting point was the Belgian Paul Otlet as founder of documentation around the turn of the 20th century. In an excursus the German chemist and information pioneer Wilhelm Ostwald is especially mentioned as part of the intellectual network of Otlet.

\section{Einleitung}

Im Sommer 2008 erschien in der Zeitschrift "Der Spiegel" ein Artikel mit der Überschrift "Vater der Zettelsuchmaschine". ${ }^{2}$ Gemeint war der Belgier Paul Otlet (1868-1944), der seit Ende des 19. Jahrhunderts versuchte, die Welt im Zettelkasten einzufangen (Abb. 1). Seine "Suchmaschine mit Zetteln" nutzte ein Hilfsmittel seiner Zeit, um das Wissen der Welt in einer "Datenbank" zu erfassen. " "The Web Time Forgot" hiess ein weiterer Beitrag in der "New York Times" über Otlet und das Mundaneum in belgischen Mons, ein Museum, das 2008 sein 10-jähriges Jubiläum in Mons gefeiert hat. ${ }^{4}$ Das Mundaneum war ebenfalls eine Idee von Otlet und wurde 1919 in Brüssel eröffnet. Das Mundaneum in Mons heute enthält u.a. die Überreste dieser Institution sowie das Archiv des Wirkens Otlets, der 1895 zusammen mit Henry La Fontaine in Brüssel das Institut International de Bibliographie gründete und die Dezimalklassifikation Melvil Deweys zur Universellen Dezimalklassifikation ausbaute.

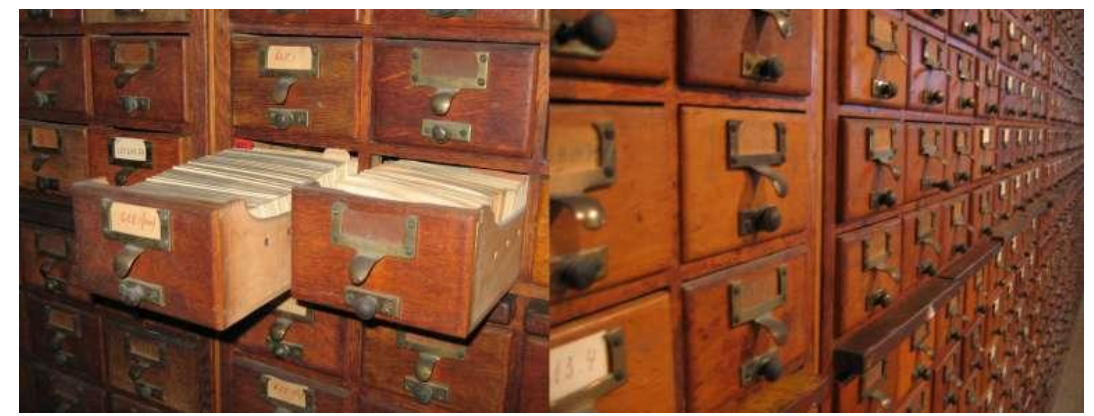

Abb. 1: Analoge Räume - Die Welt im Zettelkasten (Mundaneum, Communauté française, Mons)

Otlet gilt als Begründer der modernen Dokumentation, der heutigen Informationswissenschaft. Seine Ideen 
waren Vorläufer mancher Entwicklungen der modernen Informationsgesellschaft. Sein Mundaneum sollte neben einer Welt-Bibliothek in seinem Institut International de Bibliografie auch ein Welt-Museum, eine Welt-Universität, eine Welt-Enzyklopädie und die Vereinigung aller wissenschaftlichen Gesellschaften seiner Zeit umfassen (vgl. Abb. 2). Auch die netzartige Verknüpfung zwischen Büchern, die durch von ihm geschaffene Institutionen zu bewältigen wäre, wurde von Otlet früh thematisiert: "This development consists in establishing the connections each individual book has with all of the other books and forming from them all what might be called The Universal Book." ${ }^{5}$ Er entwickelte neben dieser Art von "Internet auf Papier" auch erste Ideen, um mit technischen Mitteln eine netzartige Kommunikation zwischen Wissenschaftlern zu ermöglichen. Zudem hatte er früh (1906) Ideen zu einer Mikroverfilmung, um die Wissensexplosion zu bewältigen.

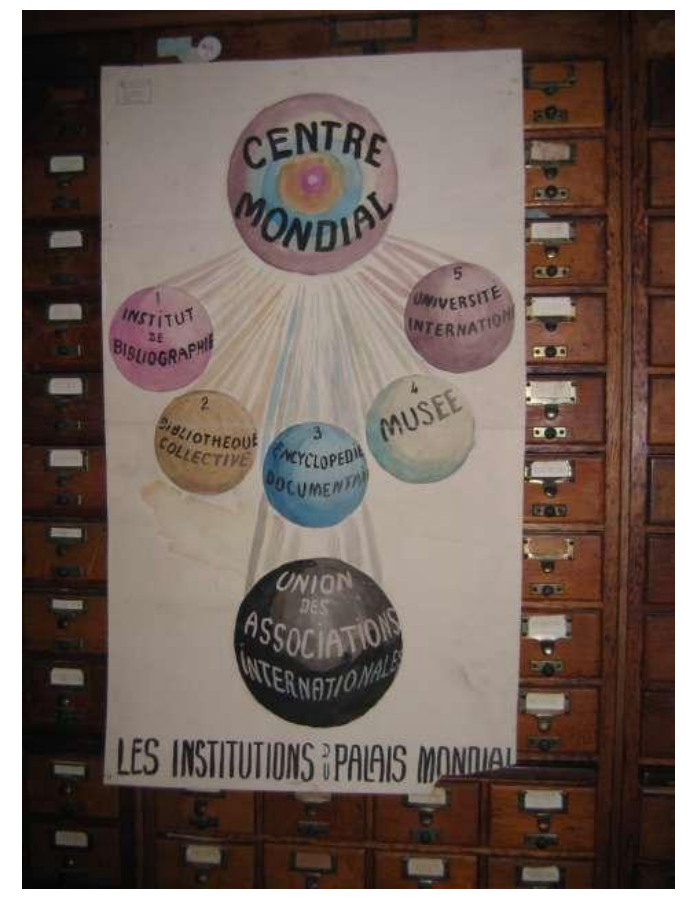

Abb. 2: Die Welt am Zettelkasten: Historisches Plakat "Centre Mondial" (Archiv, personal papers of Paul Otlet in the Mundaneum, Communauté française, Mons).

Auch der Anlass für diesen Beitrag, die Konferenz "Analogous spaces - architecture and the space of information, intellect and action", hatte Paul Otlet als Ausgangspunkt. Diese interdisziplinäre, vom 15. bis 17. Mai 2008 an der Universität von Ghent in Belgien stattgefundene Konferenz versammelte Architekten und Stadtplaner, Computerwissenschaftler, Ingenieure, Bibliothekare und Informationsspezialisten, Wissenschafts-, Bibliotheks- und Kunsthistoriker zu einem Austausch über analoge, virtuelle und digitale Räume, über Struktur, Form und Architektur von Information, Wissen, und Erinnerung und damit zusammenhängende intellektuelle und soziale Netzwerke. Das Thema der Konferenz und so auch dieser Beitrag bringen Bibliotheksbau, Informationsarchitektur und das moderne Informationswesen zusammen mit einer historischen Blick auf Ursprünge, Dissonanzen und Kontinuitäten und erfüllen damit Peter Burkes wichtigen Zweck von Geschichtsschreibung, "Entfamiliarisierung ..., eine Form der Entfremdung, die das ehemals Vertraute fremd macht und das ehemals Natürliche willkürlich." ${ }^{6}$

Die Konferenz war Teil von schon weit mehr als einem Jahrzehnt andauernden Aktivitäten, die Geschichte von Information und Kommunikation oder Informationsgeschichte als Fachgebiet zu etablieren. In Deutschland kann z.B. eine vom Wissenschaftshistoriker Christoph Meinel 1993 an der Herzog-August-Bibliothek in Wolfenbüttel durchführte Konferenz als ein Startpunkt angesehen werden. ${ }^{7}$ Immer mehr Monografien zur Informationsgeschichte werden publiziert. Letztes Jahr erschien sogar ein erstes Buch zur Informationsgeschichte als Fach. ${ }^{8}$ Dieser Beitrag kann vielleicht gleichzeitig als Literaturbericht zu aktuellen Veröffentlichungen der Informationsgeschichte angesehen werden. ${ }^{9}$

Die Geschichte wissenschaftlicher Information und Kommunikation beschäftigt sich vor allem mit der historischen Entwicklung wissenschaftlicher Publikations- und Kommunikationsmedien sowie der Geschichte ihrer Nutzung und ihrer Erschliessung in "Memory"-Institutionen (Archive, Bibliotheken, Museen) und Informationssystemen. Dazu gehört also auch die Geschichte der Bibliotheken und Medien. Die Geschichte wissenschaftlicher Information und Kommunikation ist Teil der Kulturgeschichte. Sie ist zudem Teil einer umfassend verstandenen Wissenschafts- und Technikgeschichte und schließt die Entwicklung von 
Informationstechnologien ein. In der Wissenschafts- und Technikgeschichte selbst sind als Folge einer ,kulturellen Wende' Kommunikationsprozesse und Werkzeuge wissenschaftlicher Forschung als Forschungsgegenstände in den letzten Jahren zunehmend thematisiert worden. Wissenschaftliche Disziplinen (Fachkulturen) werden als soziale und intellektuelle Infrastruktur der Wissenschaft gesehen, wo Aushandlungsprozesse und Macht und damit Kommunikationsprozesse entscheidend sind. Wissenschaft als kulturelles Phänomen wahrzunehmen bedeutet, "die historische Dimension des Wissens und seiner Repräsentationsformen, seiner grundlegenden Kategorien und Medien, seiner Praktiken und kulturellen, sozialen und ökonomischen Verwebungen ernst zu nehmen". ${ }^{10}$ Damit ist die Bedeutung einer Geschichte wissenschaftlicher Information sichtbar. Gerade die Entwicklung des Internets und der modernen Informationsund Kommunikationstechnologien stellt althergebrachte Vorstellungen von Begriffen, wie z. B. Dokument, Format, Publikation, Zeitschrift, Sammlung oder Bibliothek, in Frage und verbindet damit die zukünftige Entwicklung mit der Überlieferung.

\section{Die Konferenz “Analogous Spaces"}

\subsection{Zur Vorgeschichte der Konferenz}

Die Konferenz “Analogous Spaces” in Ghent war Treffpunkt für eine Gemeinschaft von Wissenschaftlern, die sich das erste Mal 2002 im Mundaneum in Mons auf einem Workshop getroffen hatte. Dieser trug den Titel "Architecture of Knowledge: the Mundaneum and European Antecedents of the World Wide Web". ${ }^{11}$ Direkter Vorläufer der Veranstaltung in Ghent war eine Konferenz 2005 in Urbana-Champaign, Illinois mit dem Titel "European modernism and the information society : informing the present, understanding the past". ${ }^{2}$ Ziel dieser und der Konferenz in Mons war der Austausch über Forschungsergebnisse zu einer Gruppe europäischer Gelehrter. Deren Aktivitäten sollten mit den von ihnen besonders in der ersten Hälfte des 20. Jahrhunderts gegründeten Institutionen dazu dienen, verfügbares Wissen und veröffentlichte Information zu sammeln, zu organisieren und auszutauschen. Neben Beitragen zu Otlet gab es auf diesen Treffen solche zu Zeitgenossen wie den Stadtplaner und Biologen Patrick Geddes (1854 - 1932), den Philosophen Otto Neurath (1882-1945), den Schriftsteller H.G. Wells (1966-1946), die französische Dokumentarin Suzanne Briet (1894-1989) sowie den Chemiker und Nobelpreisträger Wilhelm Ostwald (1853-1932).

Alle diesen Namen verbinden Gedankengänge und begriffliche Schwerpunkte, die selbst aus heutiger Sicht modern klingen. Dazu gehören die Umwandlung von Wissen durch Repräsentation, Fragmentierung und Visualisierung, die Architektur von Wissen und Information, der Netzwerk-Gedanke, die Bedeutung von Metadaten und Standardisierung, die Diskussion um Zentralisierung oder Dezentralisierung von Information, die Frage des Verhältnisses von Information zum Lernen oder zur Werbung, der Einsatz maschineller Hilfsmittel und Werkzeuge (Medien) zur Verarbeitung und Verbreitung von Information und zur Informationsbewältigung, das Problem zwischen Wissenschaft und Öffentlichkeit sowie die Internationalisierung und Globalisierung.

Ein weiteres Ziel der Tagungen war es ausserdem, die bisherige Tradition der historischen Arbeiten zum Informationswesen, wie sie sich in den Sammelbänden der beiden 1998 und 2002 stattgefundenen Konferenzen zur Geschichte wissenschaftlich-technischer Informationssysteme und in einem 1998 herausgegebenen Sammelband widerspiegelt, ${ }^{13}$ interdisziplinär zu erweitern. So gab es in Urbana-Champaign Vorträge aus den Disziplinen Architektur, Bibliotheksgeschichte, Medientheorie und -geschichte, Soziologie und Wissenschaftsphilosophie sowie dem Bereich Kulturwissenschaften. Die Konferenz in Ghent erweiterte nun dieses Spektrum noch einmal.

\subsection{Das Thema der Konferenz}

In seiner Einleitung zum ersten Konferenztag gab der belgische Wissenschaftler und Mitveranstalter Pieter Uyttenhove (Universität Ghent) eine erste Beschreibung seiner Vorstellungen von "Analogen Räumen”, die sich darauf beziehen, "dass jede Wissenschaft oder jedes Wissen, jeder Gedanke, jede Erinnerung, jede Aktion sich ihren eigenen 'Raum' schafft und diese Räume oft sehr ähnlich zu Strukturen der Architektur organisiert sind." Deutlich wird dies nicht zuletzt an Begriffen aus dem Computer- und Informationsbereich wie Software- oder Informations-Architektur. Auch Information und Wissen hat Struktur, Form und Architektur und die Grundfrage der Konferenz ist die nach Ähnlichkeiten und Analogien zwischen materiell oder virtuell sich manifestierendem Wissen und dessen Aufenthaltsorten wie Publikationsmedien, Bibliotheken und Datenbanken. 


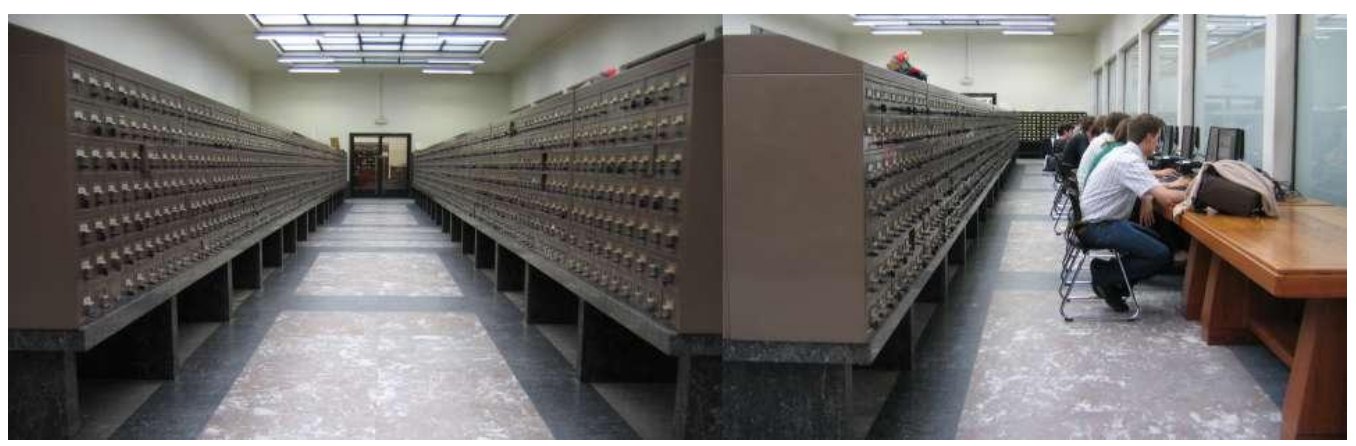

Abb. 3: Analoge Räume - Alte und neue Kataloge in der Universitätsbibliothek Ghent

Das Thema "Analoge Räume" lässt sich nach zwei Richtungen hin differenzieren: Vom Begriffspaar

Analog/Digital her unterscheiden sich z.B. physische Räume für die Aufbewahrung von Information und Wissen in gedruckten Medien, z.B. reale Bibliotheken, von digitalen Räumen der elektronischen Bibliothek. So ist das Miteinander alter und moderner Bibliothekskataloge in der Ghenter Universitätsbibliothek wunderbar sichtbar gewesen (Abb. 3). Gleichzeitig lassen sich auch Analogien und Repräsentationen zu diesen analogen und digitalen Räumen thematisieren. Im Rahmen der Konferenz ging es um analoge Beziehungen zwischen Räumen, in denen Wissen geschaffen, gesammelt, geordnet, verteilt und genutzt wird. Mögliche Typen von Räumen eines umfassenden Raumbegriffes, die im "Call for Papers" zur Konferenz erwähnt wurden (vgl. auch http://www.analogousspaces.com/Overview), waren reale Räume des Wissens und deren Architektur (Museen, Bibliotheken, Städte, Infrastrukturen, Warenhäuser, ...), Räume um Daten und Informationen zu sammeln (Datenbanken, Suchmaschinen, das System wissenschaftlicher Kommunikation, ...), das Dokument oder Buch als Raum des Wissens sowie dessen Architektur (Inhalt, Layout, Typographie, ...), aber auch die Repräsentation von Räumen in Form von Diagrammen, Organisations-Schemata, Visualisierungen, Klassifikationssystemen, usw. Architektur diente bei all diesen Räumen oft als Mittel und Metapher.

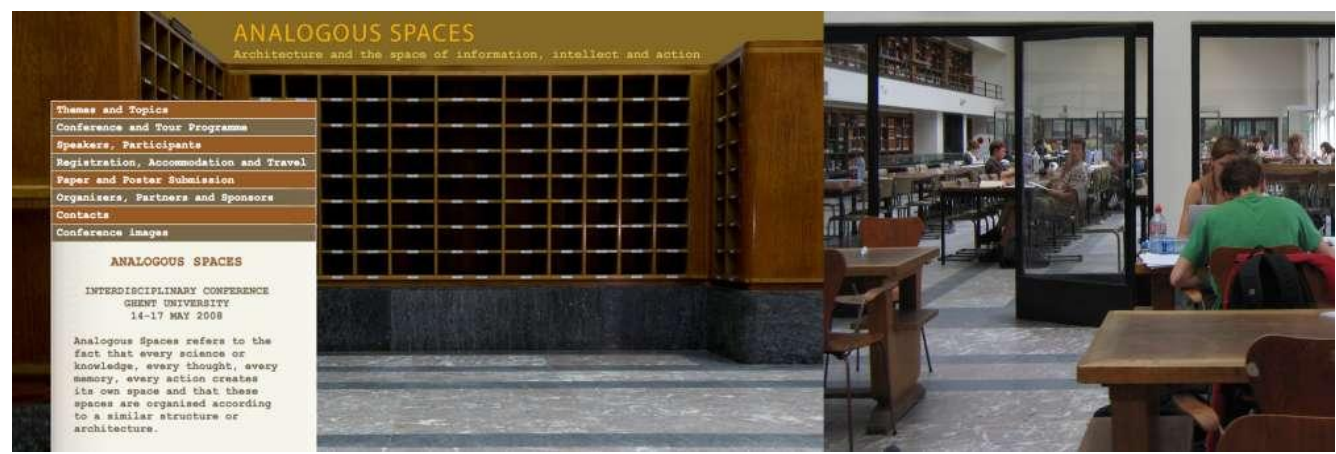

Abb. 4: Analoge Räume - Konferenz-Website "Analogous Spaces" und Lesesaal der Universitätsbibliothek Ghent

Im modernen Informationswesen ist Informationsarchitektur als Begriff und Fachgebiet etabliert. ${ }^{14}$ Ein Buch-Klassiker zum Thema definiert Informationsarchitektur so:

"1. The structured design of shared information environments

2. The combination of organization, labelling, search, and navigation systems within web sites and intranets 3. The art and science of changing information production and experiences to support usability and findability 4. An emerging discipline and community of practice focussed on bringing principles of design and architecture to the digital landscape" 15

Das Konferenzthema ist sicher auch als Teil des sogenannten "Spatial Turn" der Geistes-, Kultur- und Sozialwissenschaften zu sehen. ${ }^{16}$ "So ermöglicht das epistemologische Potential der Raumkategorie, 'wissenschaftliches Wissen nicht mehr im abstrakten Raum von Begriffs- und Ideengeschichte zu thematisieren, sondern es in seiner Kontingenz und lokalen Situiertheit, im historischen Prozess seiner Produktion darzustellen."' ${ }^{17}$ Der Reiz des Konferenzthemas lag einerseits in seiner Vagheit und andererseits in seiner interdisziplinären Stoßrichtung. Die oft auch visuell sehr ansprechenden Präsentationen, die in diesem Beitrag nicht alle erwähnt werden können, sind in großen Teilen auf der Konferenz-Website verfügbar (http://www.analogousspaces.com - vgl. Abb. 4) und bieten vielfältige Anregungen, Themen weiter zu denken bzw. zu neu entwickeln. 


\subsection{Der Ort der Konferenz}

Warum trafen sich die an Otlet Interessierten nun gerade in Ghent zu einer Konferenz über “Analoge Räume”? Hierfür können mehrere Gründe genannt werden.

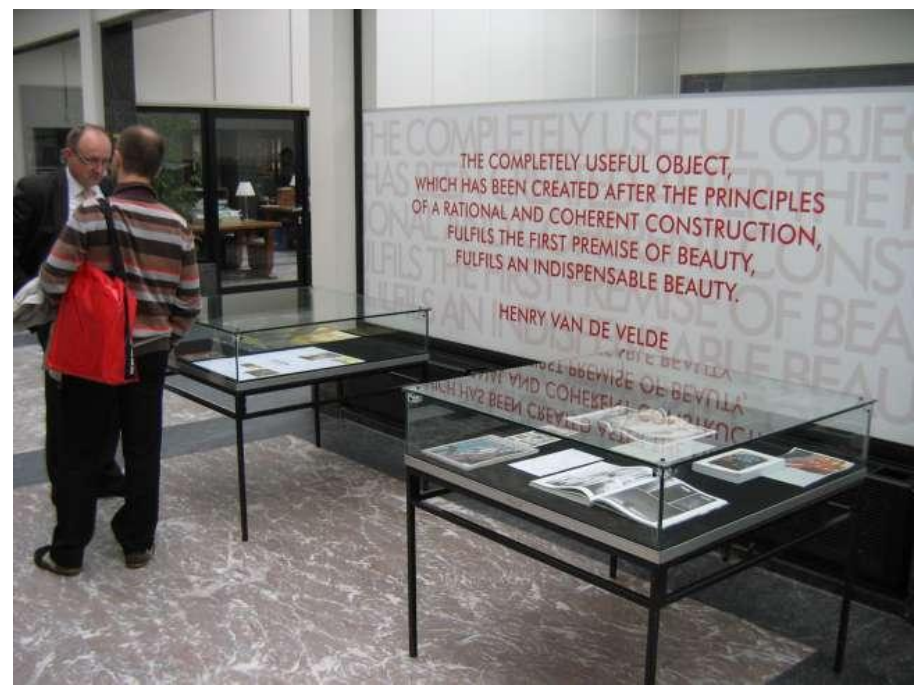

Abb. 5: Im Eingangsbereich der Universitätsbibliothek Ghent im Mai 2008

1) Die Universitätsbibliothek Ghent (http://lib.ugent.be/index_en.html ), in Person ihrer Direktorin Sylvia Van Peteghem Mitveranstalterin der Konferenz, setzt einen besonderen Schwerpunkt in der Architektur und arbeitet dabei mit lokalen Wissenschaftlern im Bereich Architektur und Städtebau zusammen. Gerade Otlet hat durch seine Zusammenarbeit mit Le Corbusier und durch seine zeitweisen auch stadtplanerischen Aktivtäten Interesse aus diesen Bereichen geweckt. Auch der Eröffnungsvortrag am Vorabend der eigentlichen Konferenz wurde vom Office for Metropolitan Architecture (OMA - http://www.oma.nl), einem Architekturbüro, das von Rem Koolhaas geleitet wird, bestritten. Im Bibliotheksbereich ist das Büro durch den Bau der Seattle Public Library besonders bekannt geworden. Das Bibliotheksgebäude in Ghent selbst ist in den dreißiger und vierziger Jahren des letzten Jahrhunderts nach Plänen des Architekten Henry van de Velde (1863-1957) gebaut worden (Abb. 5). Die Bibliothek wurde auf dem höchsten Punkt der malerischen flämischen Stadt Ghent gebaut, und das Magazin wurde als $64 \mathrm{~m}$ hoher Bücherturm ("Book Tower") konzipiert (vgl. Abb. 6).

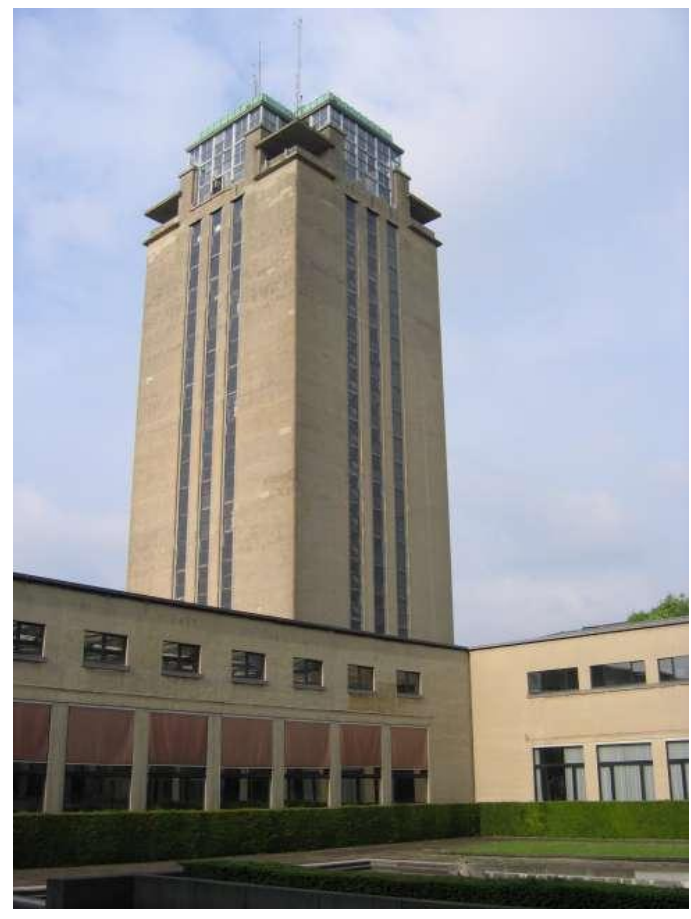

Abb. 6: Der Bücherturm der Universitätsbibliothek Ghent 
2) Die 1817 gegründete Universität Ghent hatte von der Stadt die Stadtbibliothek übernommen, die ihrerseits aus einer Schulbibliothek entstanden war. Leiter der Bibliothek in der Zeit von 1869 bis 1911 war Ferdinand Vander Haeghen (1830-1913), der eher als archivarischer Sammler denn als Universitätsbibliothekar agierte. Vander Haeghen kann ebenfalls als Vorläufer und Teil der unten (Abschnitt 2.4.) erwähnten bibliografischen Bewegung an der Wende zum 20. Jahrhundert gesehen werden. Er selbst, der auch Kontakt zu Otlet hatte, war allerdings eher ein Kritiker der Otletschen Aktivitäten. ${ }^{18}$ Vander Haeghen bearbeitete eine Bibliographie Ghenter Verlage und baute eine umfassende Sammlung von ephemeren Schrifttum mit Bezug zu Ghent auf. Sie enthielt verschiedenste gedruckte oder geschriebene Dokumente etwa Kleindrucksachen des Alltagsbereiches wie Fahrkarten, Geschäftsdrucksachen, Rundschreiben, Zeitungsausschnitte, Prospekte, Werbeschriften und anderes, was häufig nur weggeworfen wurde und wird. Heute stellt diese damals wohl von vielen als überflüssig angesehene Sammlung einen einzigartigen Schatz zur Lokalgeschichte von Ghent dar.

3) Nicht zuletzt umfasst der Titel der Konferenz neben dem analogen Raum auch den digitalen Raum als Analogon. Und auch hier ist die Universitätsbibliothek Ghent, obwohl als Ursprung kaum bekannt, weltweit wegweisend an der Entwicklung und Gestaltung der heutigen, modernen Informationslandschaft beteiligt gewesen, stammt doch Herbert Van den Sompel aus Ghent und hat dort von 1981 bis 1998 die EDV-Abteilung der Universitätsbibliothek geleitet. Van den Sompel (vgl. http://public.lanl.gov/herbertv/) entwickelte Ideen für die kontextsensitive und dynamische Verlinkung von Internetquellen, die mit SFX einen der ersten Linkresolver als Ergebnis hatten. Diese ermöglichen im Bereich digitaler Bibliotheken die direkte Verfügbarkeit von Dokumenten.

Eigentlicher Veranstaltungsort der durch knapp 100 Teilnehmer besuchten Konferenz war dann aber das Museum der schönen Künste in Ghent (http://www.mskgent.be).

\subsection{Paul Otlet als Ausgangspunkt und heimlicher Spiritis rector der Konferenz}

Mittelpunkt vieler Beiträge und eigentlicher Ausgangspunkt der Konferenz war der Belgier Paul Otlet, ${ }^{19}$ der Ende des 19. Jahrhunderts damit begann, eigene Ideen zur Organisation wissenschaftlicher Kommunikation zu entwickeln. Gerade um die Wende zum 20. Jahrhunderts führte das immense Wachstums der Zahl wissenschaftlicher Publikationen sowie die steigende Bedeutung der naturwissenschaftlichen und technischen Literatur auf internationaler Ebene zur Bildung einer bibliographischen oder auch Bibliotheks- und Dokumentationsbewegung, eines intellektuellen Netzwerkes, dem neben Otlet auch Henri La Fontaine, Wilhelm Ostwald und andere angehörten. Viele der damals entwickelten Ideen können als Vorläufer von wichtigen Prinzipien und Kennzeichen der modernen Informationsgesellschaft gesehen werden (vgl. Abschnitt 2.1).

Das Ziel war die Rationalisierung und Industrialisierung des Umgangs mit wissenschaftlicher Information. Ein erstes berühmtes Beispiel im 19. Jahrhundert waren die Aktivitäten der britischen Royal Society zum Royal Society Catalogue of Scientific Papers und der seit Anfang des 20. Jahrhunderts entstandene International Catalog of Scientific Literature. 1895 gründeten die Belgier Paul Otlet und Henri La Fontaine in Brüssel das Institut International de Bibliografie (IIB), das auf Grundlage der 1876 vom Amerikaner Melvil Dewey entwickelten Dezimalklassifikation Nachweise zur wissenschaftlichen Literaturproduktion der Welt sammeln sollte. Mit ihrer europäischen Variante der Dezimalklassifikation, der Universellen Dezimalklassifikation (UDC), reagierten Otlet und La Fontaine auf das immense Anwachsen der immer spezialisierter werdenden wissenschaftlichen Literatur in Form von Zeitschriftenaufsätzen. In den dreissiger Jahren schrieb Otlet mit einem "Buch über das Buch" sowie einem Buch über "die Welt" zwei Werke, die sein Denken zusammenfassten. ${ }^{20}$ Die Dezimalklassifikation wurde von Dewey primär als Aufstellungssystematik in Bibliotheken vorgeschlagen. Auch eine offizielle deutsche Version der Dewey-Dezimalklassifikation (DDC) existiert seit 2005. Die DDC beruht wie die UDC auf einer Einteilung aller Gebiete des menschlichen Wissens unter Verwendung von Ziffern und Sonderzeichen und wird noch heute vielfältig weltweit verwendet. Vor kurzem richtig gestellt wurde, dass die Ursprünge der Deweyschen Dezimalklassifikation wohl nicht, wie oft beschrieben, auf Leibniz zurückgehen. $^{21}$ Die oberste Ebene der Klassifikation von 0 bis 9 wird auf der nächsten Stufe wiederum durch zehn Ziffern unterteilt. Ein Beispiel für eine UDC-Notation 622.33:338.974 "Wirtschaftskrisen im Kohlenbergbau" zeigt, dass bei der UDC durch den Doppelpunkt mehrere Fachthemen miteinander kombiniert werden konnten. 


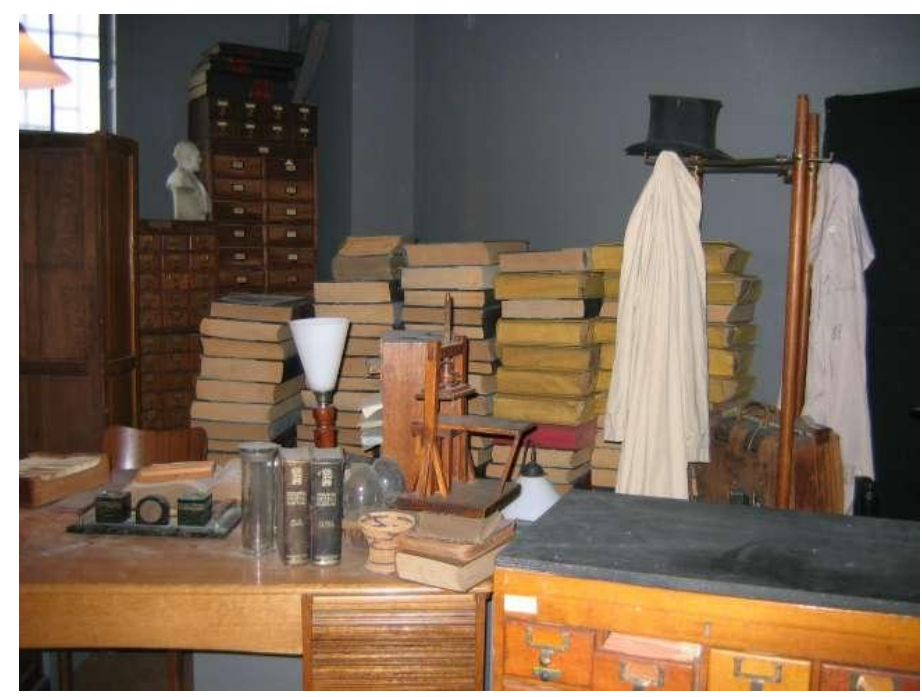

Abb.7: Schreibtisch von Otlet im Mundaneum in Mons

(Archiv, personal papers of Paul Otlet in the Mundaneum, Communauté française, Mons)

Am ersten Konferenztag stellte der als eine Art Keimzelle aller Aktivitäten zu Otlet fungierende Otlet-Biograph W. Boyd Rayward Orte und Gesichter des intellektuellen Netzwerkes um Otlet vor, wobei er besonders auf frühe Einflüsse auf Otlet in der Zeit der "Belle époque" einging. Die Leiterin des Archivs des Mundaneums in Mons, Stéphanie Manfroid, zeigte räumliche Bezugspunkte aus den Otlet'schen Beständen in Mons (vgl. Abb. 7).

Weitere Beiträge zu Otlet kamen vom Belgier Steffen Ducheyne ("Otlet: Ontology, epistemology, and the circle of knowledge") 22 und vom deutschen Verwaltungswissenschaftler Stefan Fisch ("Files and books. Paul Otlet and the beginnings of the International Institute of Administrative Sciences (IIAS-IISA) at Brussels"). Der letzte Beitrag weist auf die Geschichte eines verwaltungstechnischen Umganges mit Information hin, wie er in Organisationen der staatlichen, kirchlichen oder gelehrten Verwaltung auftritt und in einem aktuellen Buch für die frühe Neuzeit detaillierter behandelt wird. ${ }^{23}$

Ergänzt wurden die Beiträge zu Otlet am 2. Tag durch den amerikanischen Informationswissenschaftler Michael Buckland, der in seinem Keynote-Vortrag darauf hinwies, dass neben Otlet auch weitere Personen der Informations- und Bibliotheksgeschichte für die Tagungsthemen von Relevanz sind. Dabei erwähnte er folgende Personen:

- den deutschen Bibliothekar Martin Schrettinger (1772-1851), der vor 200 Jahren den Begriff Bibliothekswissenschaft als erster nutzte und für Bibliotheken eine systematische Aufstellung forderte,

- den amerikanischen Bibliothekar Melvil Dewey (1851-1931), der die systematische Aufstellung nach der von ihm entwickelten Dezimalklassifikation propagierte,

- den lettisch-deutschen Chemie-Nobelpreisträger Wilhelm Ostwald, der die auch geographische Isolation von Wissenschaftlern durch "Brücken" verbinden wollte und zugunsten räumlicher Effizienz einheitliche Formate für Druckwerke einführte (vgl. Abschnitt 3. dieses Aufsatzes),

- den deutsch-jüdischen Fotochemiker Emanuel Goldberg (1881-1970), der durch Mikrotechniken der Fotographie und - als erster - gleichzeitigen elektronischen Retrieval in Mikroformaten Distanzen zwischen Forschenden und ihren Medien überbrückte, ${ }^{24}$

- die französische Dokumentarin Suzanne Briet (1894-1989), die durch die Plazierung einer Antilope in einen Zoo dieser die Bedeutung eines Dokumentes gab, ${ }^{25}$

- den englischen Informationswissenschaftler Robert Fairthorne (1904-2000), der das Ordnen von Objekten, um diese in Retrievalsystemen suchbar zu machen, als "marking" und "parking" beschrieb.

Buckland wies darauf hin, dass Analogien als konstruktive und erläuternde, auf selektiver Ähnlichkeit beruhende Bilder stets kulturell und situativ bedingt sowie konventionell und zweckbestimmt verwendet werden. Auch Ereignisse wie diese Konferenz schaffen Raum z.B. für angeregte Diskussionen. Besonders gefallen hat dem Autor dieses Berichtes die von Buckland verwendete Analogie und Metapher für Informationsspezialisten als Jagdhund, die von Suzanne Briet stammt ("a hunter's dog, guided, guiding"). ${ }^{26}$

Neben dem gemeinsamen Ausgangspunkt Otlet wurden an drei Konferenztagen jeweils ein Schwerpunkt "analoger Räume" behandelt. Aus der Sicht des Autors humorvolle Beobachtungen am Rande unterstützten das Tagungsthema oder betrafen den Terminus Zeit-"Raum". So kam es bei manchen der Vorträge gelegentlich, wenn besonders umfangreiche Applikationen vorgestellt wurden, zu Meldungen vom benutzten Rechner wie 
"Low disk space?" oder "You are moving out of space". Bei einer Präsentation passierte Letzteres dem Vortragenden mit der Zeit. Ein Hinwies von der Moderation wurde mit der Frage "Wieviel Minuten?" erwidert. Die Antwort "Zwei" führte zu einem nachdenklichen Schweigen des Präsentierenden von gut einer Minute, bevor dieser mit einer längeren Zusammenfassung schloss. Auch Zeit hatte also einen Raum auf der Konferenz. So hatten sich diejenigen, die ein Poster eingereicht hatten, der Herausforderung zu stellen, ihren Beitrag in genau 3 Minuten dem Plenum vorzustellen.

\subsection{Räume von intellektuellen Netzwerken}

Am Beispiel des Begründers des Faches Wissenschaftsgeschichte, des Belgiers George Sarton, der auch Kontakt mit Wilhelm Ostwald hatte, suchten der Belgier Christophe Verbruggen und der amerikanische Wissenschaftshistoriker und Sarton-Biograph Lewis Pyenson ${ }^{27}$ nach Verbindungen zwischen intellektuellen Netzwerken mit sozialen Bewegungen wie Sozialismus und Pazifismus, denen z.B. auch Otlet und Henri La Fontaine angehörten. Daniel Laquas Thema war die Transnationalität intellektueller Kooperationen. Gerade Belgien ist bis heute eine Nation, die eine Fülle transnationaler Konferenzen und Organisationen beherbergte (vgl. auch Abb. 16 am Schluss dieses Beitrages).

Thomas Soetens und Kora Van den Bulcke von Workspace Unlimited (http://www.workspace-unlimited.org) stellten in einer Präsentation via Skype ihr mobiles und experimentelles Labor zur kreativen Produktion neuer Medien vor. Kunst, Architektur und die digitale Technik versuchen hier neue Kontexte in hybrider und erweiterter Realität zu schaffen. Der niederländische Historiker für moderne Architektur Koos Bosma zeigte in einer wunderschönen Präsentation mit dem Titel "In search for DataSpace" sein Analogieverständnis zwischen Daten- und realen Räumen.

\subsection{Räume von Wissen und Erinnerung}

In seiner Einführung zum zweiten Tag führte der Mitorganisator der Konferenz Wouter van Acker an, dass alle Informationspraktiken Raum erzeugen. Räum nach Descartes sind leere Behälter, im Leibnizschen Sinne sind Räume immer von einem Körper besetzt. Visualisierung von Wissen zur Information schafft Räume, wie sie z.B. die Pläne von U-Bahn-Linien in Großstädten darstellen. Reale Räume werden andererseits auch durch Visualisierung zu Wissen. Der zweite Konferenztag hatte also die Analogie realer und virtueller Räume des Wissens im Blickpunkt, klassischerweise z.B. die Bibliothek. So berichtet der britische Bibliotheks- und Informationshistoriker Alistair Black, der heute an der University of Illinois in Urbana-Champaign lehrt, über die Entwicklung der Gebäude von öffentlichen Bibliotheken in Großbritannien, die auch Thema eines noch erscheinenden Buches sein werden. ${ }^{28}$

Klassisch war auch der in einer wunderschönen Präsentation vorgestellte Beitrag des belgischen Buchwissenschaftlers Pierre Delsaerdt zu nennen, der eine typographische Analyse von durch Christophe Plantin gedruckten Nachschlagewerken des 16. Jahrhunderts vorlegte. Ein Text wird auch im Raum der Seiten eines Buches z.B. typographisch dargestellt. Eine wichtige angesprochene Frage war hier, wie Buch-Design Erkenntnis steuern kann. ${ }^{29}$ Charles van den Heuvel erkundete in seinem Beitrag "Urban Grids, Computer Grids and Global Grids" analoge Architekturen der Informationsgesellschaft. So illustrierten oft pyramidale Darstellungen das positivistische Gedankengut der frühen Informationsbewegung. Auch der unten noch ausführlicher erwähnte Ostwald nutzte eine Pyramide in Anlehnung an Auguste Comte zur Systematisierung der Wissenschaften. Van den Heuvel zeigte, dass auch moderne Strukturen des Semantic Web positivistische Elemente enthalten (vgl. Abb. 8). 


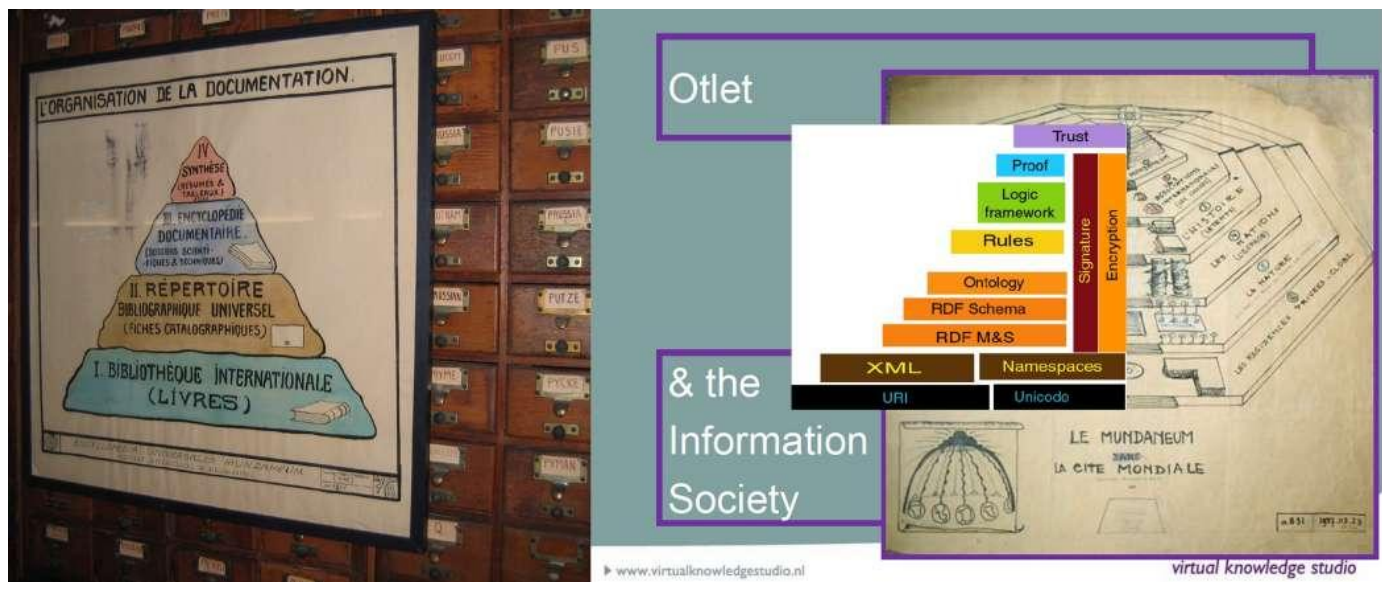

Abb. 8: Otlet, das Mundaneum und die Informationsgesellschaft

(Archiv, personal papers of Paul Otlet in the Mundaneum, Communauté française, Mons)

Reale Räume des Wissens sind auch Museen, die im Vortrag des amerikanischen Wissenschaftlers Nader Vossoughian durch seine Sicht auf Otto Neurath thematisiert wurden. Neurath, ein österreichischer dem logischen Empirismus (Wiener Kreis) nahestehender Philosoph und Entwickler einer Form von

"Bildersprache", ${ }^{30}$ wurde hier als "funny polymath" bezeichnet, was sicher auf viele der auf dieser Konferenz behandelten Personen der Geschichte zutraf. Für Neurath war ein Museum eine Art Warteraum, der im öffentlichen Raum ohne Grenzen sichtbar sein sollte, um informelles Lernen zu ermöglichen. Er konzipierte in diesem Sinne Wanderausstellungen als eine Form von Museum als Massenproduktion. 1925 betonte Neurath die Bedeutung von Werbung als Werkzeug für Bildung: "The modern advertisement will show us the way".

Die Französin Dominique Rouillard hinterfragte die Idee der universalen Universität mit Bezug zum amerikanischen Architekten, Designer und Philosophen Buckminster Fuller. Weitere Vorträge reflektierten die Realität moderner virtueller Räume. Philippe Colombet präsentierte Google Books. Tamar Sadeh stellte die vielfältigen Dimensionen der Darstellung von Suchergebnissen nach einer Recherche z.B. in Drill-Down-Menüs dar.

\subsection{Räume des Handelns und der Entscheidung}

Der dritte thematische Block der Konferenz bezog sich vom Konzept her auf den Raum Stadt und damit in Richtung Stadtplanung. Städte können ja als Systeme von Informationsarchitektur angesehen werden. Architektur meint hier nicht das Design von Gebäuden sondern Systemarchitektur, also die Interaktion von Komponenten eines komplexen Systems. ${ }^{31}$ Aber auch der Bezug zum wirtschaftlichen Wissensmanagement war vom Call for Papers her möglich. Voll treffend war hier der Beitrag des Amerikaners Jesse Lecavalier mit dem Titel "Wal-Scrapers. Extra-Medium, Supra-Medium, Infra-Medium". Anhand des Organisationshandelns des Konzerns Wal-Mart zeigte Lecavalier, dass Logistik durchaus als neue Grundlagenwissenschaft der modernen Informationsgesellschaft angesehen werden kann. Dass z.B. Bibliotheken und ihre Organisationsherausforderungen hinsichtlich der Verwaltung und Nutzung von Information durchaus mit Supermärkten vergleichbar sind, verdeutlichen auch im Bibliothekswesen gern verwendete Metaphern wie "One-Stop-Shop". 32

Dieser Konferenzteil startete mit zwei Vorträgen zu Information und Wissen sowie zu den Ausprägungen des Begriffes "Dokument" aus der Sicht der Informationtechnik und Computerwissenschaften. Begriffe wie Information, Kommunikation, Medien und Wissen als vielfältig verwendete Schlüsselbegriffe unserer Zeit fordern ja immer wieder philosophisch-theoretische Reflexionen heraus. Charakteristisch für das Konzept Information ist für den Autor, dass seine Realität plausibel und nützlich in Abhängigkeit von der Fachdisziplin in vielfältiger, oft widersprüchlicher Art und Weise bestimmt werden kann. Information kann nie isoliert von seinen verwandten Begriffen wie Kommunikation, Wissen usw. betrachtet werden. Der Ghenter Wissenschaftler Guy de Tré zeigte dann allerdings rein technologische Lösungen für die Stufen der klassischen Pyramide von Daten, Information und Wissen auf. Wissen ensteht bei ihm z.B. aufgrund von "Data Mining". Der Vortrag des Elektrotechnikers Patrick Bergmans mit dem Titel "The new document: digital, polymorphic, ubiquitous and actionable" rekapitulierte Dokument-Modelle in den Computerwissenschaften nach Inhalt, Struktur und Format. So können die Teile von "intelligenten" Netzdokumenten heute durchaus an unterschiedlichen Orten gespeichert sein und bei Aufruf dynamisch generiert werden. Bergmans ging aber naturgemäß nicht auf den Forschungsstand in den Informationswissenschaften ein. ${ }^{33}$ 
Der Beitrag mit dem Titel "The evolution of knowledge landscapes - measurement, visualization, models and simulations" von Andrea Scharnhorst, Virtual Knowledge Studio Amsterdam

(http://www.virtualknowledgestudio.nl) schlug eine Verbindung zu den intellektuellen Netzwerken des ersten Konferenztages. Sie demonstrierte, wie die Modellierung und Visualisierung von "Wissenslandschaften" Hilfen für Entscheidungen geben können, was dann durchaus zum dritten Block passte. Heutzutage ist die Kombination von Wissensressourcen und Kompetenzen notwendig, um in komplexen Entscheidungsprozessen Problemlösungsstrategien zu entwickeln. Hilfreich können hier "Wissenslandschaften", die aufgrund der Nutzung bibliometrischer Methoden generiert werden, eine Orientierung bieten.

\section{Ein Poster und ein Exkurs: Wilhelm Ostwald und die Informationsgesellschaft}

Im Rahmen der Poster-Session auf der Konferenz stellte der Autor dieses Beitrages ein Poster vor. Es hatte den Titel "Combinatorics and order as a foundation of creativity, information organization and art in the work of Wilhelm Ostwald". ${ }^{4}$ Ostwald hatte Kontakt zu Otlet und ähnliche Ideen für Aktivitäten zum Informationswesen seiner Zeit wie dieser.

Aufgrund seiner zahlreichen Aktivitäten im Bereich des wissenschaftlichen Publizierens - dem Verfassen von Lehrbüchern, Aufsätzen, Buchreviews und Briefen sowie der Herausgabe von Zeitschriften - war sich der Chemiker und Nobelpreisträger Wilhelm Ostwald (1853-1932) ${ }^{35}$, seit 1887 Professor für Physikalische Chemie in Leipzig, von Anfang an des Problems der Informationsbewältigung bewusst. Er war einer der ersten Pioniere des deutschen Informationswesens. ${ }^{36}$ Seine Konzepte zur "Organisation geistiger Arbeit", also zur Organisation der wissenschaftlichen Kommunikation und Information, basierten auf der von ihm gesehenen Notwendigkeit, aufgrund der wachsenden Informationsflut die wissenschaftliche Kommunikation zu rationalisieren und zu vereinheitlichen. Seine Ordnungsideen, ausgeführt in einer Theorie der Ordnung innerhalb seiner Naturphilosophie, wurden von Ostwald u.a. in den Feldern der Sprache (Internationale Hilfssprache), der Papierformate, dem System wissenschaftlicher Disziplinen (in einer Pyramide der Wissenschaften ${ }^{37}$ nach dem Vorbild Auguste Comtes) sowie der Farbenlehre angewandt. Sein Vorschlag zur Normung der Papierformate ("Weltformat") wurde später mit leichten Änderungen als deutscher und internationaler Standard eingeführt (A4 etc.). Wie Frederick Winslow Taylor die industrielle Produktion verwissenschaftlichen wollte, richteten sich Ostwalds Bestrebungen auf die Verwissenschaftlichung der Produktion wissenschaftlicher Erkenntnisse. Sein sogenannter energetischer Imperativ, "Vergeude keine Energie, verwerte sie", war dabei eine wichtige Voraussetzung. Ostwald entwickelte zudem eine Theorie der Farben und der Formen, was ihn in Kontakt zu Bewegungen wie dem Deutschen Werkbund, dem Bauhaus und der niederländischen De Stijl brachte. Das Poster wies insbesondere auf Analogien zwischen In-formation und Form also Kunst hin (vgl. Abb. 9). 


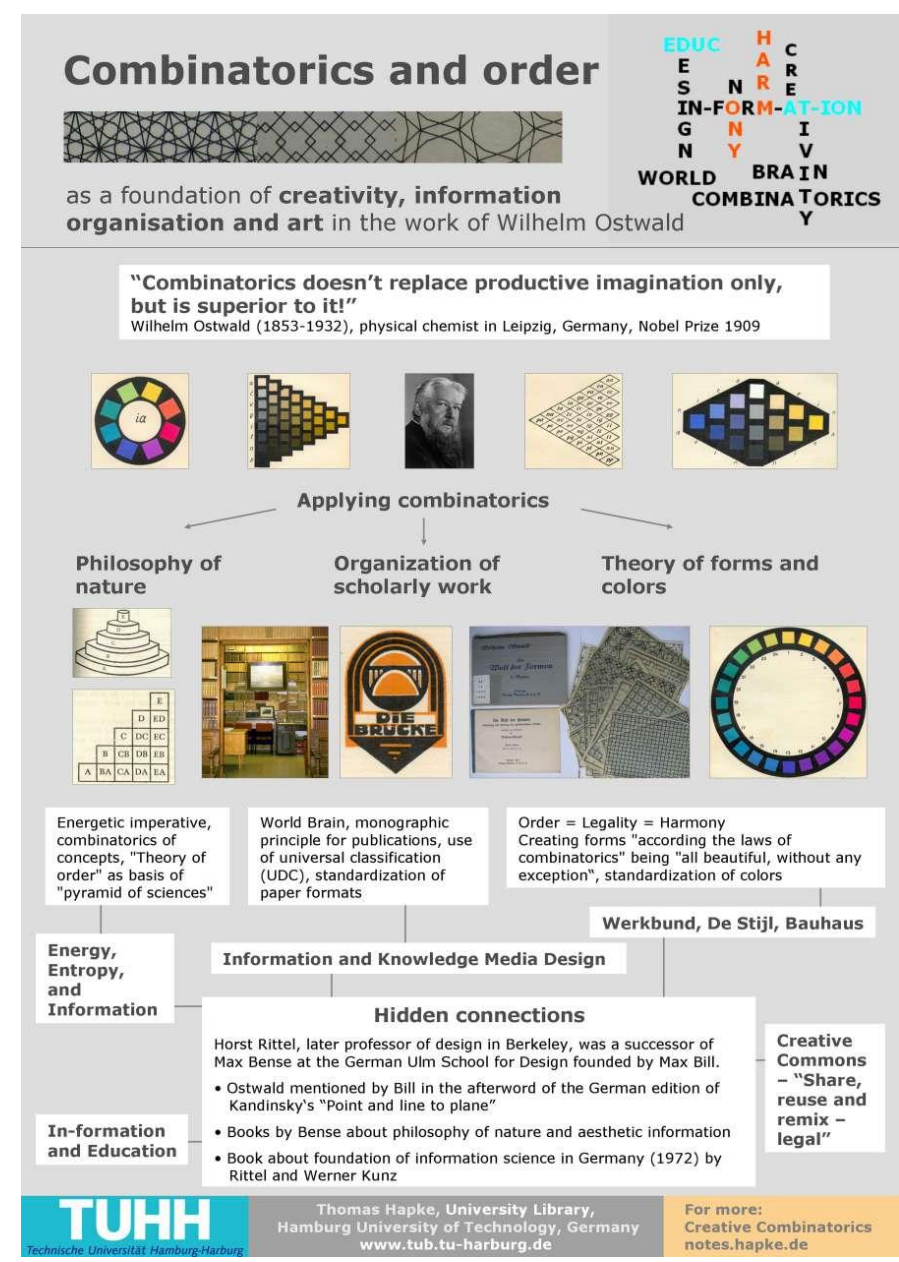

Abb. 9: Poster des Autors

Ostwald gründete zusammen mit dem Schweizer Karl Wilhelm Bührer 1911 in München die "Brücke", ein "Institut für die Organisierung der geistigen Arbeit" ${ }^{38}$ mit ähnlichen Zielen wie Otlets Institut Internationale de Bibliographie (IIB). Die Brücke sollte als Vermittlungs- und Auskunftsstelle die verstreuten Aktivitäten anderer Organisationen wie Bibliotheken, Museen, wissenschaftlichen Gesellschaften usw. verbinden. Ostwald bezeichnete die Brücke auch als "Gehirn der Welt". ${ }^{39}$ Ziel war es, auf arbeitsteiliger Grundlage eine Art Weltenzyklopädie auf Karteikarten aufzubauen. Die Organisation geistiger Arbeit sollte automatisch erfolgen, durch die allgemeine Einführung standardisierter Kommunikationsmittel - das monographische Prinzip, standardisierte Papierformate sowie Klassifikationsvermerke mit der Dezimalklassifikation für alle Publikationen. Ergebnis wäre dann als "Brückenarchiv" so etwas wie eine "umfassende, illustrierte Welt-Enzyklopädie auf Karten mit standardisiertem Format." 


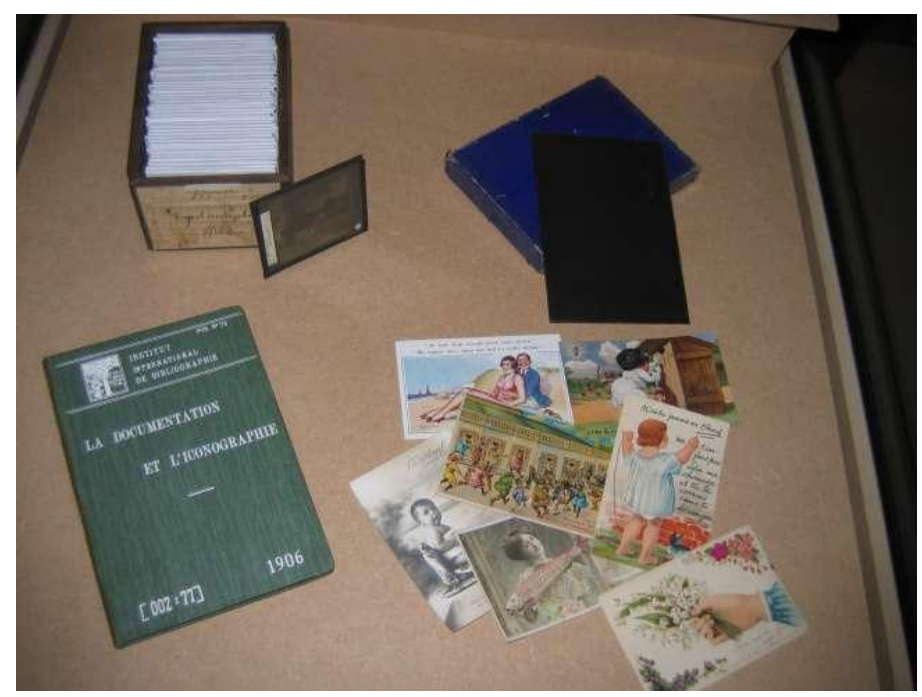

Abb. 10: Das Sammeln von Bildern als Aufgabe der Dokumentation

(Archiv, personal papers of Paul Otlet in the Mundaneum, Communauté française, Mons)

In einem 1946 erschienenen anonymen Artikel in der Zeitschrift Nature mit dem Titel "Rationalization of the literature of scientific research" 40 wird Ostwald als Vorläufer von Überlegungen zum Ersatz der wissenschaftlichen Zeitschrift genannt. In Erkenntnis der wachsenden Fragmentierung von Wissen schlug Ostwald u. a. vor, die wissenschaftliche Zeitschrift aufzulösen und statt dessen nur die einzelnen Artikel zu verschicken, da kein Wissenschaftler alle Aufsätze eines Zeitschriftenbandes lese. Diese "technische" Zerstückelung des Wissens musste durch ein einheitliches System der Ordnung in Form einer Klassifikation, der Dezimalklassifikation, zusammengehalten werden. Sein "Prinzip der unabhängigen Handhabung des einzelnen Stückes"41 oder "Monographieprinzip", hatte Ostwald schon implizit seit 1889 bei der Herausgabe seiner "Klassiker der exakten Wissenschaften" angewandt.

Das Monographieprinzip selbst, das Otlet später ebenfalls propagierte, wurde auch in der Vorgeschichte der Brücke praktiziert, hatte doch Karl Bührer 1905 die sogenannte "Internationale Monogesellschaft" gegründet. Das Unternehmen veröffentlichte sogenannte "Monos", kleine Karten oder Broschüren in standardisiertem Format. Monos waren so etwas wie die vielen "Reklamebilder", die damals in Deutschland populär waren, z.B. von den Firma Liebig. Auch das Institut International de Bibliografie von Otlet verschrieb sich der Sammlung von Bildern (vgl. Abb. 10). Bührer hatte schon früh auf die Nutzung von Karteikarten im Geschäftsleben aber auch zur Erleichterung des wissenschaftlichen Arbeitens hingewiesen: "[j]edes Blatt [dient ...] ganz konsequent nur für eine Notiz, welche oben rechts durch Anbringung eines Titels sofort rubriziert wird [...] Gelehrten ist dieses Zettelsystem zum Sammeln und Ordnen ihrer Exzerpte ganz besonders zu empfehlen." 42

Für viele frühe "Informations-Institutionen" waren das Sammeln, das Organisieren, die Werbung und die Bildung konstitutionelle Komponenten der Vermittlung von Information, wobei insbesondere die letzten beiden Komponenten bis in unsere Tage nicht immer bewusst als Teil der Vermittlungs- und Informationstätigkeit wahrgenommen werden. ${ }^{43}$ Wilhelm Ostwald entwickelte eine Art Theorie der Medien oder Kommunikationsmittel, er nannte sie "Verkehrsmittel". Diese helfen dem Gedächtnis oder der intellektuellen Arbeit durch Organisation. Ein Notizbuch oder ein Karteikarten-Index war für Wilhelm Ostwald eine "geistige Maschine". Ein Buch wurde von ihm als ein Energie-"Transformator für die Erzeugung geistiger Werte" aufgefasst. ${ }^{44}$ Auch Paul Otlet sah das Buch als "Maschine", die "Denk-Energie"übermittelt. ${ }^{45}$

"Die Kombinatorik ersetzt nicht nur die schaffende Phantasie, sondern ist ihr überlegen." schrieb Ostwald im Jahre 1929. Kreativität umfasste für Ostwald also auch "Kombinatorik". Einfälle und neue Entdeckungen seien oft nur "eine neuartige Zusammenstellung vorhandener Bestandteile". 46 Dabei wird Kombinatorik bei Ostwald nicht unbedingt in einem rein mathematischen Sinne (als Bestimmung möglicher Anordnungen unterscheidbarer oder nicht unterscheidbarer Objekte unter eventueller Berücksichtigung der Reihenfolge) sondern oft auch nur metaphorisch gebraucht! Ostwalds Auffassung von Kombinatorik als Komponente von Kretaivität findet man wieder in modernen Ideen eines alternativen Umgangs mit dem Urheberrecht im Rahmen im weltweiten Datennetz. Diese Lizenz-Modelle, die sogenannten "Creative Commons"-Lizenzen, betonen nach dem Motto "Share, Remix, Reuse — Legally " die damit mögliche Förderung von Kreativität.

Die Kombinatorik bei Ostwald ist vor allem auch innerhalb seiner pädagogischen Aktivitäten zu verstehen. ${ }^{47}$ Aktuelle bibliothekspädagogische Themen wie Informations- und Medienkompetenz als Teil lebenslangen 
Lernens schließen durchaus an Zitate von Ostwald an wie: "Dabei genügt es nicht, etwa öffentliche Bibliotheken zu gründen; es muss auch durch Vorträge und Merkhefte die Technik der Benutzung vorhandener

Bücherschätze, die gar nicht so einfach ist, den Bildungssuchenden geläufig gemacht werden."48 Für den Autor dieses Beitrages ist neben der oben genannten Analogie zwischen Information und Kunst besonders die Analogie zwischen Information, auch gelesen als "In-Formation", und Bildung bzw. Erziehung wichtig. ${ }^{49}$ Aber auch Fachgebiete wie Informationsdesign und "Knowledge Media Design" ${ }^{50}$ belegen die Beziehungen zwischen Information und Kunst bzw. Design, ebenso wie moderne Entwicklungen zur Ästhetik der Information. Einer der Exponenten der Informationsästhetik in den sechziger Jahren des letzten Jahrhunderts, der Stuttgarter Philosoph Max Bense, schrieb übrigens ähnlich wie Ostwald Bücher zur Naturphilosophie, bevor er an der vom Schweizer Max Bill ${ }^{51}$ gegründeten Ulmer "Hochschule für Gestaltung" arbeitete. Auch der Nachfolger von Bense in Ulm, Horst Rittel, später Professor für Design in Berkeley, steht für diese kombinatorisch-assoziative Beziehung zwischen Information und Design. ${ }^{52} 1972$ publizierte Horst Rittel zusammen mit Werner Kunz ein Buch zur Grundlegung der Informationswissenschaften in Deutschland. ${ }^{53}$

\section{Nachbereitung: Eine Rundreise durch Belgien}

Die sich an die Konferenz anschließende Rundreise durch Belgien führte dann zu realen Räumen der Informationsgeschichte, und zwar zu Orten, an denen das Wirken Otlets mehr oder weniger sichtbar ist und die oft auch frühe Werke des belgischen Jugendstils etwa Henry van der Veldes umfassen.

Das erste Reiseziel war die von Otlet zusammen mit dem belgischen Stadtplaner und Architekten Octave van Rysselberghe geplante Siedlung Westende an der Nordseeküste südlich von Ostende. Hier wurden ein Museum, die Villa Les Zéphyrs besucht - mit Inventar geschaffen von Van der Velde - und das ebenfalls von Rysselberghe gebaute Grand Hotel Belle Vue.

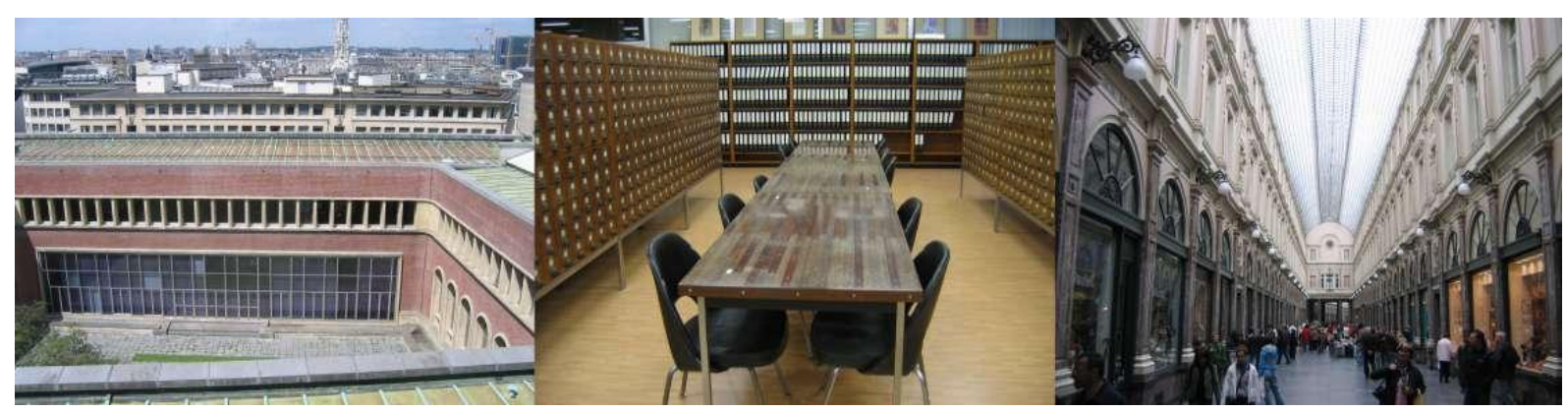

Abb. 11: Analoge Räume: Gebäude sowie Katalog in der Koninklijke Bibliotheek van België und "Les Galeries St. Hubert" in Brüssel

Am nächsten Tag trafen sich die Otlet-Touristen in Brüssel. Bei einem Besuch des Autors in der Nationalbibliothek Belgiens (KBR - http://www.kbr.be) war die optische Analogie zwischen Bibliotheksgebäude und Katalogen auffallend (vgl. Abb. 11). Gleichzeitig erinnerte eine Einkaufspassage ganz in der Nähe an analoge Räume in der Universitätsbibliothek Ghent (vgl. Abb. 3) und nahm damit die oben erwähnte Analogie zwischen Bibliotheken und Verkaufsinstitutionen und Warenhäusern wieder auf. Auch der neu gestaltete Eingangsbereich der KBR (Abb. 12) lebte von der analogen Darstellung der Welt als Bibliothek und der Bibliothek als Welt, wie sie auch das Otletsche Mundaneum symbolisiert. 


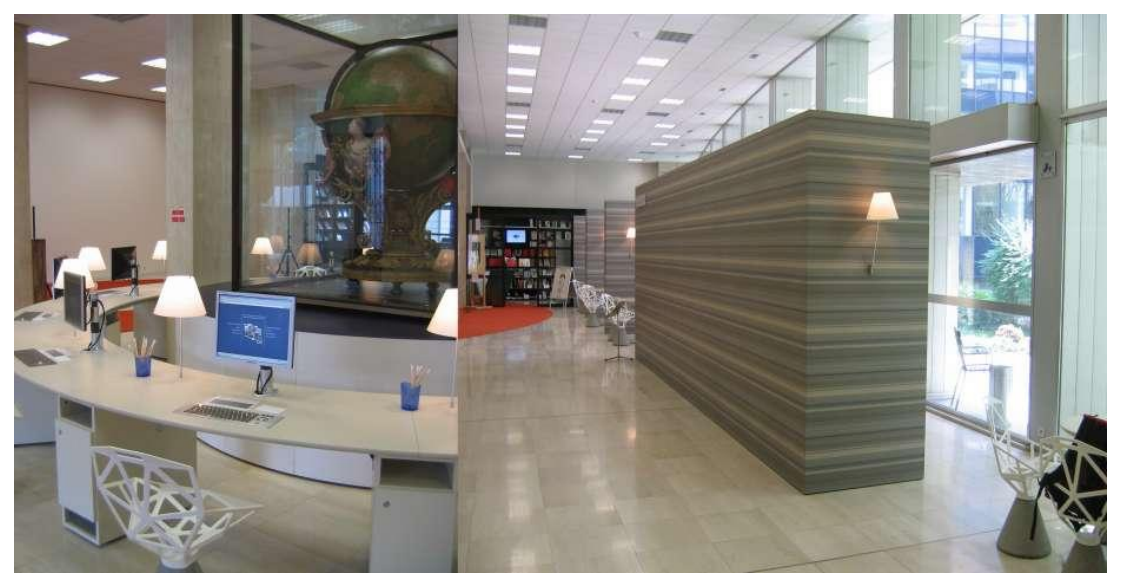

Abb. 12: Der neu gestaltete Eingangsbereich der Belgischen Nationalbibliothek: Die Welt als Analogie

Das von Otlet in Brüssel bewohnte Jugendstil-Haus (vgl. Abb. 13), das sogenannte "Hotel Otlet" (13 rue de Florence), wurde von Octave van Rysselberghe gebaut, die innere Raumgestaltung hatte Henri van der Felde übernommen. Es wird heute als Rechtsanwalts-Kanzlei genutzt.

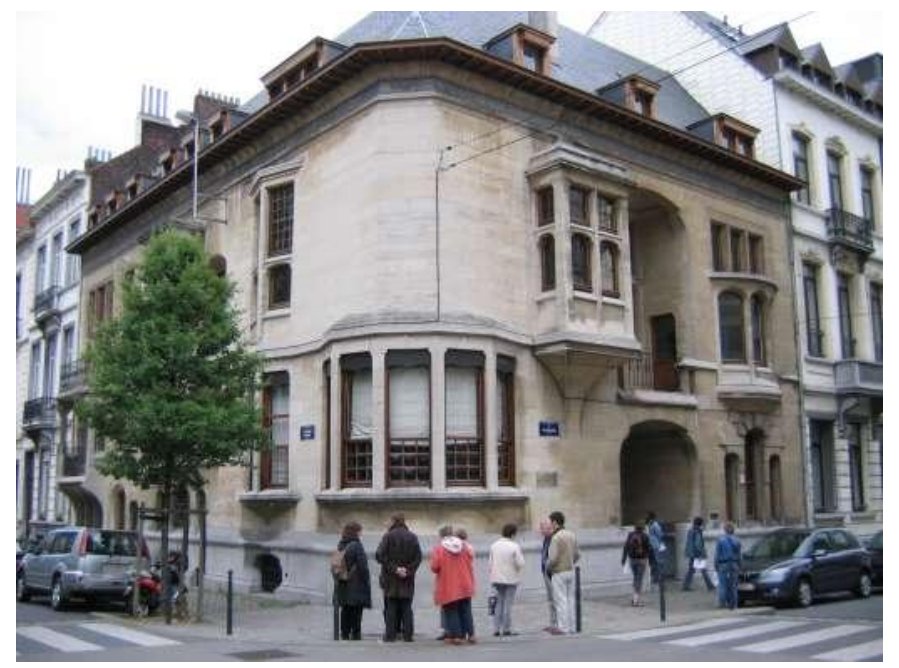

Abb. 13: Hotel Otlet

Die 1902 von Van der Velde und Constant Bosmans gebaute Bibliothèque Solvay im Parc Léopold (137 rue Belliard, vgl. Abb. 14), eines der ersten "Science Parks", war als Institut für Soziologie der Brüsseler Universität gebaut worden, finanziert durch den Chemie-Industriellen Ernest Solvay, der auch mit Ostwald in Verbindung stand. 1994 wurde sie nach mehr als zehnjährigen Verfall restauriert und steht nun als Veranstaltungsraum zur Verfügung (http://www.bibliothequesolvay.be).

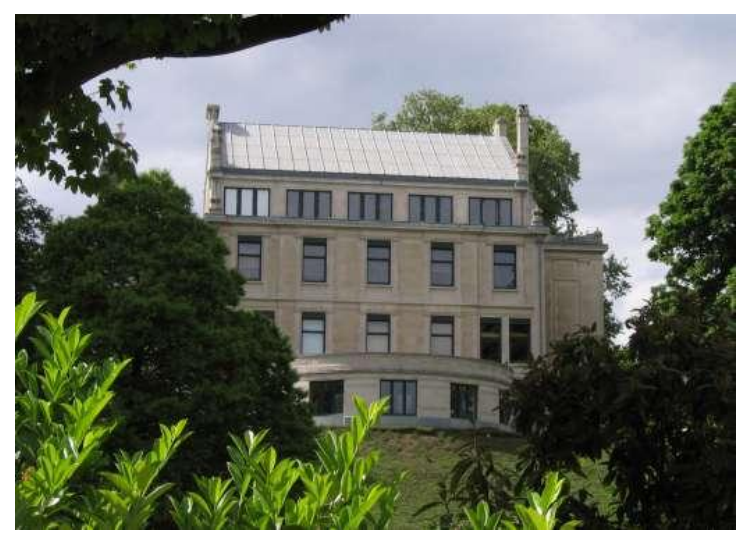

Abb. 14: Bibliothèque Solvay im Parc Léopold in Brüssel 
Am Schluss der Reise durch Belgien stand für den Autor ein Besuch und zweitägiger Forschungsaufenthalt im anfangs erwähnte Mundaneum in Mons (Abb. 15 - http://www.mundaneum.be). Otlet hatte Le Corbusier inspiriert, konkrete, aber nie verwirklichte Pläne für ein Mundaneum in Genf zu entwickeln, als ein internationales Zentrum für Information, Bildung und Wissenschaft.

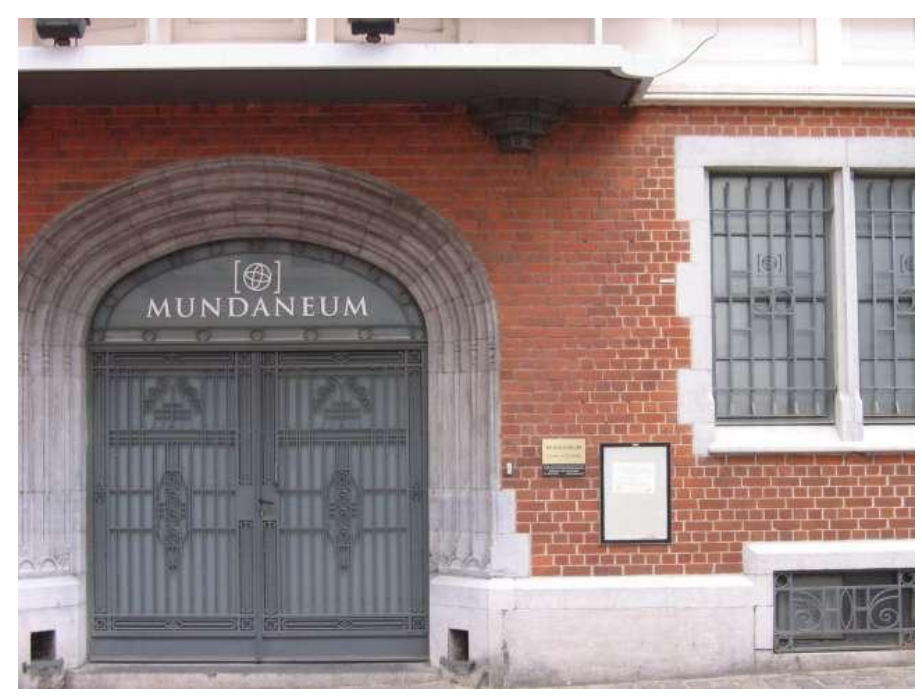

Abb. 15: Eingang zum Mundaneum in Mons

Für den Autor war der Besuch in Mons gleichzeitig eine Entdeckungsreise zum in realen Papieren niedergelegten intellektuellen Netzwerk der bibliografischen Bewegung. Lange Jahre waren die in der Dissertation von Paul Schneiders ${ }^{54}$ beschriebenen Beziehungen zwischen Paul Otlet und dem Vorgänger der von Ostwald mitbegründeten Brücke, der Internationalen Monogesellschaft, im Archiv nicht zugreifbar und verschollen. Der Briefwechsel zwischen Otlet und Karl Wilhelm Bührer, aber auch die intensiven, langjährigen brieflichen Beziehungen zwischen Otlet bzw. Lafontaine und dem deutschen Julius Hanauer, einem der Hauptbefürworter der Dezimalklassifikation in Deutschland und ebenfalls langjährigem Briefpartner Ostwalds, konnten erstmals eingesehen werden. Auch das Auffinden von Briefen zwischen Wilhelm Ostwald und Otlet gehörte zum Ertrag des Besuches in Mons. Im Ostwald-Nachlass im Archiv der Berlin-Brandenburgischen Akademie der Wissenschaften hatte der Autor bisher keine Briefe zwischen Ostwald und Otlet finden können. Deutlich wurde, dass deutsche Aktivisten durchaus Teil des Otletschen Netzwerkes und der bibliographischen Bewegung ihrer Zeit waren.

Schon 1908 hatte Ostwald, der später ja auch die Analogie der "Brücke" nutzte (vgl. Abb. 16), ähnlich wie Otlet von einem "Netz" nicht nur der Wissenschaften und den Beziehungen der wissenschaftliche Erkenntnisse untereinander, sondern auch von einem Netz der Wissenschaftler gesprochen: "Ebenso geht es nun auch mit dem einzelnen Menschen. Wenn der Kreis seines Wissens auch noch so beschränkt ist, es ist immer ein Teil jenes großen Netzes und hat daher die Beschaffenheit, daß sich die anderen Teile ohne weiteres anschließen lassen, sobald sie in das Bewusstsein und die Kenntnis des einzelnen gelangen. Wer einmal auf solche Weise in den Kreis der Wissenschaft eingetreten ist, gewinnt ähnliche Vorteile, wie der, der den Anschluß an das Telefonnetz seines Wohnortes hat. [...] So hat denn auch der Anfänger in der Wissenschaft bereits mit dem elementarsten Unterricht, den er in der Schule, von seinen Eltern, ja vielleicht nur durch persönliche Erfahrungen in seiner Umgebung aufgenommen hat, einen oder einige Fäden jenes gewaltigen Netzes erfaßt [...] Und dieses Netz hat die wertvolle, ja köstliche Beschaffenheit, daß es dasselbe ist, welches die größten und umfassendsten Geister der Menschheit untereinander verbindet." 55 


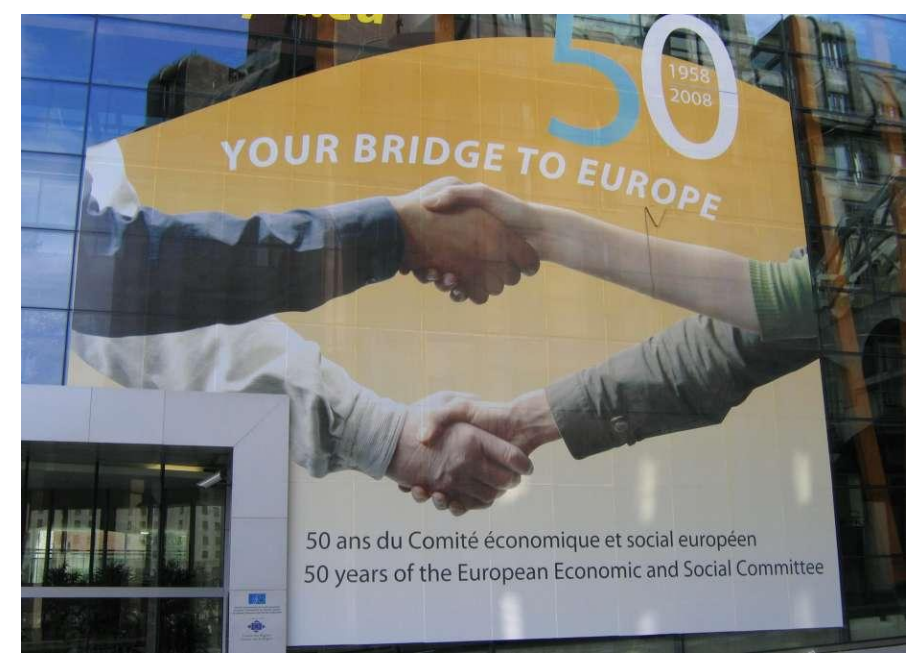

Abb. 16: Brücken (und Netze) verbinden (im Europäischen Viertel von Brüssel).

\section{Anschrift des Verfassers}

Thomas Hapke

Fachreferent für Verfahrenstechnik und Chemie

Universitätsbibliothek der TU Hamburg-Harburg

Denickestr. 22, 21073 Hamburg

http://www.tu-harburg.de/b/hapke/

\footnotetext{
${ }^{1}$ Der Autor dankt Stéphanie Manfroid, Responsable des Archives, und dem Mundaneum, 15 rue des Passages, 7000 Mons, Belgien für die Erlaubnis die Abbildungen 1, 2, 7, 8 und 10 in diesem Aufsatz zu benutzen. Alle weiteren Rechte behält sich das Mundaneum vor. Sylvia Van Peteghem, Leiterin der Universitätsbibliothek Ghent, gestattete die Veröffentlichung der Fotos 3, 4 und 5. Charles van den Heuvel, The Virtual Knowledge Studio Amsterdam, Niederlande, gebührt Dank für die Erlaubnis, eine Folie aus seinem Vortrag mit dem Titel "Urban Grids, Computer Grids and Global Grids : an historical exploration of analogous architectures of the information society" in Abb. 8 zu benutzen. Alle Fotos und die Montagen stammen vom Autor.

${ }^{2}$ Schmundt, H.: Vater der Zettelsuchmaschine. - In: Der Spiegel Nr. 26. 23.06.2008, S. 140.

${ }^{3}$ Vgl. zu Suchmaschinen aus kulturhistorischer Sicht auch: Gugerli, D.: Suchmaschinen : die Welt als Datenbank. - Frankfurt a. M.: Suhrkamp 2009.

${ }^{4}$ Wright, A.: The Web Time Forgot. - In: The New York Times, June 17, 2008. Online http://www.nytimes.com/2008/06/17/science/17mund.html . Vgl. auch die Jubiläumsschrift des Mundaneums: Le Mundaneum : les archives de la connaissance. Bruxelles: Les Impressions Nouvells 2008.

${ }^{5}$ Otlet, P.: Transformations in the bibliographical apparatus of the sciences (1918). - In: Rayward, W.B. (Hrsg.): International organisation and dissemination of knowledge : selected essays of Paul Otlet. S. 148-156. Amsterdam: Elsevier, 1990. S. 154. Online unter http://hdl.handle.net/2142/8645 .

${ }^{6}$ Burke, P.: Papier und Marktgeschrei : die Geburt der Wissensgesellschaft. - Berlin: Wagenbach, 2001. S. 10.

${ }^{7}$ Vgl. den Konferenzband: Meinel, C. (Hrsg.): Fachschrifttum, Bibliothek und Naturwissenschaft im 19. und 20. Jahrhundert. - Wiesbaden: Harrassowitz, 1997 (Wolfenbütteler Schriften zur Geschichte des Buchwesens ; 27). Siehe auch den Aufsatz Hapke, T.: Auf dem Weg zu einer Geschichte der naturwissenschaftlichen Publikation. In: Auskunft 14 (1994) S. 48-52.

${ }^{8}$ Vgl. Weller, T.: Information history : an introduction ; exploring an emergent field. - Oxford: Chandos 2008. Weitere Buchhinweise zur Geschichte des Informationswesens sind unter http://www.librarything.de/catalog/info-history zu finden. Ein Buch des Autor des oben genannten Artikels in der New York Times ist mit dabei: Wright, A.: Glut : mastering information through the ages. - Washington, DC : Joseph Henry Press 2007.

${ }^{9}$ Er ergänzt damit den Beitrag von Hapke, T.: Ein Baustein zur Geschichte wissenschaftlicher Information und Dokumentation. Eine Rezension (Elke Behrends: Technisch-wissenschaftliche Dokumentation in Deutschland von 1900 bis 1945 : unter besonderer Berücksichtigung des Verhältnisses von Bibliothek und Dokumentation) und ein Literaturüberblick - In: Auskunft 18(1998) S. 193-199. Vgl. auch folgende Reviews: Burke C.: History of information science. - In: Annual review of information science and technology 41 (2007) S. 3-53 und Black A.: Information history. - In: Annual review of information science and technology 40 (2006) S. 441 -473 sowie den Weblog des Autors: http://blog.hapke.de/?cat=5
} 
${ }^{10}$ Hagner, M.: Ansichten der Wissenschaftsgeschichte. - In: Hagner, M. (Hrsg.): Ansichten der

Wissenschaftsgeschichte. S. 7-39. Frankfurt a. M.: Fischer 2001. Hier S. 30

${ }^{11}$ Vgl. Rayward, W.B.: Knowledge organisation and a new world polity: the rise and fall and rise of the ideas of Paul Otlet. - In: Transnational Associations 55 (2003) S. 4-15 sowie van den Heuvel, C.; Rayward, W.B.;

Uyttenhove, P.: Savoir et décrire - L'architecture du savoir : une recherche sur le Mundaneum et les précurseurs européens de l'Internet. - In: Transnational Associations 55 (2003) S. 16-28.

${ }^{12}$ Die Vorträge der Konferenz sind mittlerweile als Buch erschienen: Rayward, W.B. (Hrsg.): European modernism and the information society : informing the present, understanding the past. - Aldershot, Hants.: Ashgate 2008.

${ }^{13}$ Vgl. Bowden, M.E.; Hahn, T.B.; Williams, R.V. (Hrsg.): Proceedings of the 1998 Conference on the History and Heritage of Science Information Systems : [held 23 - 25 October 1998, in Pittsburgh, Pennsylvania]. Medford, NJ : Information Today 1999, online unter http://www.chemheritage.org/explore/ASIS_documents/ASIS98_main.htm ; Rayward, W.B.; Bowden, M.E. (Hrsg.): The History and Heritage of Scientific and Technological Information Systems: Proceedings of the 2002 Conference. - Medford, NJ: Information Today 2004, online unter

http://www.chemheritage.org/pubs/asist2002/proceedings.html und Hahn, T.B.; Buckland, M. (Hrsg.): Historical studies of information science. - Medford, NJ: Information Today 1998.

${ }^{14}$ Vgl. Jacob, E.K.; Loehrlein, A.: Information architecture. - In: Annual review of information science and technology 43 (2009) S. 147-185.

${ }^{15}$ Morville, P.; Rosenfeld, L.: Information architecture for the World Wide Web. 3. ed. - Beijing: O'Reilly 2007, zitiert von Jacob, \& Loehrlein, S. 153.

${ }^{16}$ Vgl. Döring, J.; Thielmann, T. (Hrsg.): Spatial turn : das Raumparadigma in den Kultur- und Sozialwissenschaften. - Bielefeld : Transcript 2008 oder Günzel, S.: Raumwissenschaften. - Frankfurt am Main : Suhrkamp, 2009.

${ }^{17}$ Bachmann-Medick, D.: Cultural turns: Neuorientierungen in den Kulturwissenschaften. 2.Aufl. Reinbek: Rowohlt 2007, S. 314. Das eingebettete Zitat stammt aus Rheinberger, H.-J. (Hrsg.): Räume des Wissens : Repräsentation, Codierung, Spur. - Berlin : Akad.-Verl. 1997. S. 8.

${ }^{18}$ Vgl. Uyttenhove, P.; Van Peteghem, S.: Ferdinand van der Haeghen's shadow on Otlet: European resistance to the americanized modernism of the Office International de Bibliographie. - In: W. Boyd Rayward (Hrsg.): European modernism and the information society : informing the present, understanding the past, S. 89-104. Aldershot, Hants.: Ashgate, 2008.

${ }^{19}$ Es gibt zwei Biografien zu Otlet: Levie, F.: L' homme qui voulait classer le monde : Paul Otlet et le Mundaneum. - Bruxelles : Les Impressions Nouvelles 2006; sowie: Rayward, W.B.: The universe of information : the work of Paul Otlet for documentation and international organisation. - Moscow : All-Union Inst. for Scientific and Techn. Information (VINITI) 1975. Online unter http://hdl.handle.net/2142/651 . Vgl. auch Hartmann, F.: Von Karteikarten zum vernetzten Hypertext-System - Paul Otlet, Architekt des Weltwissens - Aus der Frühgeschichte der Informationsgesellschaft. - In: Telepolis 29.10.2006. Online unter http://www.heise.de/tp/r4/artikel/23/23793/1.html

${ }^{20}$ Otlet, P.: Traité de documentation : le livre sur le livre ; Théorie et pratique. - Bruxelles: Palais Mondial 1934, online unter http://hdl.handle.net/1854/5612/ sowie Otlet, P.: Monde : Essai d'universalisme : connaissance du monde, sentiment du monde, action organisée et plan du monde. - Bruxelles: Editiones Mundaneum 1935, online unter http://hdl.handle.net/1854/8321

${ }^{21}$ Vgl. Waldhoff, S.: Von der rechten Administrierung des Wissensschatzes : Zu Leibniz' Entwürfen einer bibliographisch-bibliothekarischen Sachsystematik. - In: Hartbecke, K. (Hrsg.): Zwischen Fürstenwillkür und Menschheitswohl - Gottfried Wilhelm Leibniz als Bibliothekar. S. 159-241. Frankfurt a.M.: Klostermann 2008. Hier S. 163 und S. 219.

${ }^{22}$ Vgl. auch Ducheyne, S.: "To treat of the world" Paul Otlet's ontology and epistemology and the circle of knowledge. - In: Journal of Documentation 65 (2009) 2, S. 223-244.

${ }^{23}$ Vgl. Brendecke A.; Friedrich, M.; Friedrich, S. (Hrsg.).: Information in der Frühen Neuzeit : Status, Bestände, Strategien. - Berlin: LIT Verl. 2008.

${ }^{24}$ Vgl. Buckland, M.K.: Emanuel Goldberg and his knowledge machine : information, invention, and political forces. - Westport, Conn.: Libraries Unlimited 2006.

${ }^{25}$ Briet, S.: What is documentation? : English translation of the classic French text / Transl. and ed. by Ronald E. Day. - Lanham, Md.: Scarecrow Press 2006.

${ }^{26}$ Vgl. Briet, S.: Bibliothécaires et documentalistes. - In: Revue de la documentation 12 (1954) S. 41-45. Hier heisst es: "La documentation professionelle [...] doit etre à la pointe de la recherche er meme dans une certaine mesure - comme le chien du chasseur - tout à fait en avant, guidé, guidant." Die Ehefrau des Autors züchtet Jagdhunde der Rasse Welsh Springer Spaniel.

27 Pyenson, L.: The passion of George Sarton : a modern marriage and its discipline. - Philadelphia : American Philosophical Society 2007.

${ }^{28}$ Black, A.; Pepper, S.; Bagshaw, K.: Books, buildings and social engineering : early public libraries in Britain 
from past to present. - Aldershot: Ashgate 2009. Black war auch an einem weiteren schon erschienen Buch zur frühen englischen Informationsgesellschaft beteiligt: Black, A.; Muddiman, D.; Plant, H.: The early information society : information management in Britain before the computer. - Ashgate, Aldershot, 2007.

${ }^{29}$ Vgl. Enenkel, K. A. E.; Neuber, W.: Cognition and the book : typologies of formal organisation of knowledge in the printed book of the early modern period ; [papers given at the conference "Formale Erkenntnissteuerung im Buchdruck der Frühen Neuzeit... "]. - Leiden: Brill 2005.

${ }^{30}$ Vgl. zu Neurath: Vossoughian, N.: Otto Neurath : the language of the global polis. - Rotterdam: NAi Publ. 2008 sowie Hartmann, F.: Bildersprache : Otto Neurath Visualisierungen. 2.Aufl. - Wien: Univ.-Verl. 2006.

${ }^{31}$ Vgl. Coward, L.A.; Salingaros, N.A.: The information architecture of cities. - In: Journal of Information Science 30 (2004)2, S. 107-118.

${ }^{32} \mathrm{Vgl}$. auch Weinberger, D.: Das Ende der Schublade : die Macht der neuen digitalen Unordnung. - München : Hanser, 2008, der sein Buch mit einem Beispiel der Präsentation von Waren in einer Supermarktkette beginnt.

${ }^{33} \mathrm{Vgl}$. Lund, N.W.: Document theory. - In: Annual review of information science and technology 43 (2009) S. 399-432.

${ }^{34}$ Poster im Netz unter http://doku.b.tu-harburg.de/volltexte/2008/404/ ; Weblog zum Konferenzposter des Autors "Creative Combinatorics" unter http://notes.hapke.de

${ }^{35}$ Mehr zur Biografie Ostwalds bei Zott, R.: Friedrich Wilhelm Ostwald (1853-1932), nunmehr 150 Jahre jung. In: Angewandte Chemie 115 (2003), S. 4120-4127 und Kim, M.G.: Wilhelm Ostwald (1853-1932). - In: HYLE - International Journal for Philosophy of Chemistry 12 (2006) 1, S. 141-148. Online unter http://www.hyle.org/journal/issues/12-1/bio_kim.htm

${ }^{36}$ Vgl. Hapke, T.: Ordnung, Fragmentierung und Popularisierung: Wilhelm Ostwald zur wissenschaftlichen Information und Kommunikation. - In: Krug, K. (Hrsg.): Wissenschaftstheorie und -organisation : Vorträge zu dem Symposium anlässlich des 150. Geburtstages von Wilhelm Ostwald am 18. September 2003 in Großbothen. S. 63-78. - Großbothen: Wilhelm-Ostwald-Gesellschaft 2004.

37 Ostwald, W.: Die Pyramide der Wissenschaften. - Stuttgart: Cotta 1929.

${ }^{38}$ Bührer, K.W.; Saager, A.: Die Organisation der geistigen Arbeit durch "Die Brücke". - Ansbach: Seyboldt 1911.

${ }^{39}$ Vgl. Ostwald, W.: Das Gehirn der Welt. - München: Brücke 1912, und Sachsse, R.: Wilhelm Ostwald :

Farbsysteme, Gehirn der Welt. - Ostfildern-Ruit: Hatje Cantz 2004.

40157 (1946) No. 3997, S. 745-748.

${ }^{41}$ Ostwald, W.: Die chemische Literatur und die Organisation der Wissenschaft. Leipzig: Akad. Verl.-Ges. 1919. S. 96.

${ }^{42}$ Quelle: Bührer, K.W.: Ueber Zettelnotizbücher und Zettelkatalog. - In: Fernschau 4 (1890) S. 190-192. Zur

Geschichte des Ursprungs von Karteien aus Büros und Firmen in der Bibliothek vergl. Krajewski, M.:

Zettelwirtschaft : die Geburt der Kartei aus dem Geiste der Bibliothek. - Berlin: Kadmos 2002.

${ }^{43} \mathrm{Vgl}$. auch Hapke, T.: Roots of mediating information : aspects of the German information movement. - In:

W.B. Rayward (Hrsg.), European Modernism and the Information Society. Informing the Present, Understanding the Past. S. 307-327. - Aldershot: Ashgate 2008.

44 Ostwald, W.: Die Philosophie der Werte. - Leipzig: Kröner 1913. S. 342.

45 Day, R.E.: The modern invention of information. - Carbondale: Southern Illinois Univ. Press 2001. S. 15.

${ }^{46}$ Siehe Ostwald, W.: Kombinatorik und schaffende Phantasie (1929). - In: Ostwald, W.: Forschen und Nutzen : Wilhelm Ostwald zur wissenschaftlichen Arbeit / hrsg. v. G. Lotz... S. 28-30. - Berlin: Akademie-Verl.1978 (2. erw. Aufl. 1982).

47 Vgl. auch Hapke, T.: Wilhelm Ostwalds pädagogische Aktivitäten und die Ökonomisierung der Technik "geistiger Arbeit". - In: Ein Netz der Wissenschaften? Wilhelm Ostwalds "Annalen der Naturphilosophie" und die Durchsetzung wissenschaftlicher Paradigmen : Vorträge des Kolloquiums, veranstaltet von der Sächsischen Akademie der Wissenschaften zu Leipzig und dem Institut für Philosophie der Universität Leipzig im Oktober 2007 / hrsg. von Pirmin Stekeler-Weithofer, ... S. 67-97. Leipzig: Hirzel, 2009. (Abhandlungen der Sächsichen Akademie der Wissenschaften zu Leipzig, Philogisch-historische Klasse, Band 81, Heft 4, 2009) und Hapke, T.: Von der "Weltausstellung im Kleinen" zum "lebenden Lehrbuch" : Bildungsbezogene Komponenten früher Ansätze von Weltbibliotheken um 1900. - In: Rainer Koschke, Otthein Herzog, Karl-Heinz Rödiger, Marc Ronthaler (Hrsg.): INFORMATIK 2007 - Informatik trifft Logistik, Band 2. S. 485-490. - Bonn: Gesellschaft für Informatik 2007.

${ }^{48}$ Ostwald, W.: Zur Biologie des Forschers : Vortrag, gehalten während der 350. Jahresfeier der Universität Genf. - In: Actes du Jubilé de 1909 / Université de Genève. S. 114-121. - Gennève: Librairie Georg \& Cie 1910.

${ }^{49}$ Passend dazu auch das Buch von Scheibel, M.: Architektur des Wissens : Bildungsräume im Informationszeitalter. - München: Kopad 2008.

${ }^{50}$ Vgl. z.B. Stephan, P. F.: Knowledge Media Design - Konturen eines aufstrebenden Forschungs- und Praxisfeldes". - In: Eibl, M.; Reiterer, H.; Stephan, P. F.; Thissen, F. (Hrsg.), Knowledge Media Design : Theorie, Methodik, Praxis. 2., korr. Aufl., S. 1-42 - München: Oldenbourg 2006. Er erwähnt auch die Brücke und Ostwald. Siehe auch Klütsch, C.: Computer Grafik : Ästhetische Experimente zwischen zwei Kulturen : Die 
Anfänge der Computerkunst in den 1960er Jahren. - Wien: Springer 2007.

${ }^{51}$ Max Bill nennt Ostwald im Nachwort zu Kandinsky, W.: Punkt und Linie zu Fläche: Beitrag zur Analyse der malerischen Elemente. - Bern-Bümpliz: Benteli 1973. S. 210.

${ }_{52}$ Rith, C.; Dubberly, H.: Why Horst W. J. Rittel Matters. - In: Design Issues 23 (2007) 1, S. 72-91.

${ }^{53}$ Kunz W.; Rittel, H.: Die Informationswissenschaften: ihre Ansätze, Probleme, Methoden und ihr Ausbau in der Bundesrepublik Deutschland. - München: Oldenbourg 1972.

${ }^{54}$ Schneiders, P.: De bibliotheek- en documentatiebeweging 1880-1914; bibliografische ondernemingen rond 1900. Diss. - Amsterdam, Univ., Literaturwiss. Fak. 1982.

${ }^{55}$ Ostwald, W.: Grundriß der Naturphilosophie. - Leipzig: Göschen 1908. S. 15-17. 